\title{
Le stockage de gaz naturel : impact piézométrique
}

\author{
par Gilbert Meunier
}

du Gaz de France

\section{LE STOCKAGE DE GAZ NATUREL : UN ENJEU ÉCONOMIQUE CONSIDÉ- RABLE}

Le stockage de grands volumes de gaz naturel est nécessaire pour constituer un volant de réserve entre une fourniture de gaz régulière dans l'année et une consommation variable en fonction des variations climatiques, du rythme de vie et des activités des usagers : quantités supplémentaires nécessaires pendant les mois d'hiver, pointes de consommation des épisodes froids, variation intrahebdomadaire et intrajournalière. Le stockage souterrain offre une réponse particulièrement adaptée à ce besoin. Quand la consommation est inférieure à la fourniture de gaz par gazoducs et méthaniers, le gaz est injecté dans le stockage; quand la consommation est supérieure à la fourniture, le gaz est soutiré du stockage.

Disposer de stocks importants de gaz permet aussi de faire face aux risques d'incidents techniques pouvant interrompre l'acheminement du gaz depuis des gisements éloignés (Russie, Algérie, Mer du Nord, Pays-Bas).

Lors d'une interruption durable d'un approvisionnement, la réadaptation de l'offre à la demande est obtenue en utilisant au maximum les souplesses des contrats non défaillants, en effaçant la clientèle interruptible et enfin en faisant appel à un complément de soutirage des réservoirs souterrains.

ll existe en France 15 stockages souterrains de gaz naturel ; leur capacité utile était au $1^{\text {er }}$ novembre 1994 voisine de 10 milliards de $\mathrm{m}^{3}(\mathrm{n})$ de gaz, pour un stock total supérieur à 22 milliards de $\mathrm{m}^{3}(\mathrm{n})$.

Le dispositif actuel du Gaz de France comprend les réser- voirs souterrains en nappe aquifère de Beynes (Beynes Supérieur et Beynes Profond), Saint-llliers, Chémery, Cerville-Velaine, Gournay-sur-Aronde, Saint-Clair-sur-Epts, Soings-en-Sologne et Germigny-sous-Coulombs, et les stockages en cavités salines de Tersanne et Etrez. Elf Aquitaine Production exploite pour sa part les sites de Lussagnet et Izaute.

Deux sites GDF sont en phase de démarrage de l'exploitation : le réservoir en nappe aquifère de Céré-la-Ronde et le stockage en cavités salines de Manosque. Les besoins de stock utile augmentent en effet avec le développement général des consommations de gaz naturel à un rythme annuel de 3 à $4 \%$.

A cet égard, les bilans prévisionnels à moyen terme du Gaz de France font apparaitre que ce besoin sera de près de $140 \mathrm{TWh}^{*}$ dans dix ans. Le dispositif actuel sera alors largement insuffisant. Les sites existants, une fois tous développés, peuvent apporter, avec une amélioration de leurs performances, une capacité de $130 \mathrm{TWh}$ environ. Des développements supplémentaires qui ne peuvent être certifiés aujourd'hui sont encore possibles, mais en tout état de cause l'apparition du déficit ne pourra être repoussée au-delà de l'an 2000.

La mise en exploitation de nouveaux stockages est donc nécessaire. Gaz de France projette ainsi de mettre en service à la fin de la décennie deux nouveaux sites:

Le gisement de gaz de Trois-Fontaines à proximité de Saint-Dizier (Haute-Marne) sera progressivement converti en

(3) $1 \mathrm{~m}^{3}$ comespond en moyenne a $11,3 \mathrm{kWh}$.

The creation of a new natural gas storage represents a considerable economic stake. Therefore, before briging it into operation. it is important to know if all guaranties, necessary to a successfull integration in the regional subterranean enrivonment, are fulfilled. Such guaranties can be obtained through the following steps:

- Geological studies of the basin describing the system geometry.

- Research on the natural hydrodynamic functioning.

- Daten insertion and setting in a model of calculation.

- Anticipation of the possible impact in terms of variations of pression andlor of displaced water volumes. 
stockage et devrait fournir en quelques années une capacité hivernale de l'ordre de $50010^{6} \mathrm{~m}^{3}$.

Le site aquifère des Landes de Siougos ensuite, dans les niveaux dolomitiques du Dano-Paléocène de la région de Mont-de-Marsan, participera pendant plus d'une vingtaine d'années à cette augmentation globale pour devenir l'équivalent de l'important site de Chémery $\left(7\right.$ milliards de $\left.\mathrm{m}^{3}\right)$.

On comprend ici que la mise en place progressive de telles capacités tampon représente une condition indispensable au développement d'une énergie par ailleurs peu polluante, abondante et bon marché et qu'une insertion réussie dans l'environnement est une priorité pour la pérennité de cette technique.

\section{II 盢 LES IMPACTS POSSIBLES DU STOC- KAGE EN NAPPE AQUIFÈRE}

Sur le territoire français, le stockage de gaz souterrain à grande échelle, donc à des conditions économiques acceptables, ne peut se concevoir que par l'intermédiaire de 2 techniques largement éprouvées : le stockage en cavités salines creusées par dissolution dans les niveaux sédimentaires de sel et le stockage en milieux poreux dans les aquifères des deux principaux bassins sédimentaires français le Bassin Parisien et le Bassin Aquitain. Dans les deux cas apparaissent des limitations techniques, économiques et de plus en plus environnementales. Pour le cas aquifère, nous allons nous focaliser sur ces dernières, les expliciter et montrer quelles méthodologies sont mises en cuvre et quelles réponses sont apportées.

Schématiquement, le développement puis l'exploitation d'un stockage de gaz naturel est susceptible d'entraîner deux sortes de conséquences sur les niveaux concernés.

- Tout d'abord, l'injection d'une quantité importante de gaz accompagnée progressivement d'une exploitation cyclique du stockage va modifier le régime hydrodynamique des aquifères profonds (pressions et écoulements): les pressions en tête ou les niveaux d'eau des divers puits pourront évoluer avec une amplitude dépendant du niveau intercepté et de la localisation géographique des ouvrages.

Dans les zones où ces couches aquifères remontent jusqu'en surface une modification du débit naturel des sources ou, de façon moins perceptible, des rivières, est envisageable. Ainsi une partie limitée de ce qui peut être considéré comme une ressource à long terme pourra quitter son lieu de stockage naturel. Une variation du régime des pressions pourra également amener des transferts d'un niveau sur l'autre se traduisant par le mélange d'eaux de qualités différentes.

- Par ailleurs, et bien que le gaz naturel soit considéré comme très peu réactif vis-à-vis de l'eau contenue initialement dans les pores de la roche, diverses modifications physico-chimiques sont à attendre sur des périodes très longues; la principale étant la solubilisation du méthane et son transport par le mouvement naturel des nappes aquifères à échéance de plusieurs centaines de siècles.

D'autres évolutions d'importance limitée interviennent également; elles ont fait et font encore l'objet de recherches spécifiques, comme la formation de $\mathrm{H}_{2} \mathrm{~S}$ ou l'adsorption de l'odorisant THT sur les roches du sous-sol.

\section{LES CONSÉQUENCES POUR LES UTILISATEURS}

Pour diverses raisons (augmentation des consommations, pollutions de surface...) les nappes profondes sont et seront de plus en plus sollicitées par des utilisateurs diversifiés: thermalisme, géothermie, eau potable ou à usage industriel, stockage... Dans le cas du Bassin Aquitain, fortement plissé, la remontée locale jusqu'en surface des terrains profonds le long de «rides » entraine inévitablement l'usage agricole voir l'irrigation intensive.

Il devient alors nécessaire à l'occasion de tout projet industriel d'étudier la manière dont il s'insère dans le contexte hydrogéologique et de préciser les conséquences à attendre pour les autres utilisateurs.

Concernant le stockage de gaz naturel, celles-ci interviennent à différents termes. Les éventuelles conséquences physico-chimiques ne peuvent physiquement se faire sentir en dehors du périmètre de protection avant plusieurs milliers voire dizaines de milliers d'années.

Par contre la diffusion des évolutions de pression liées aux injections/soutirages se réalise à une échelle de temps beaucoup plus courte : quelques mois à quelques années pour des distances de quelques dizaines de kilomètres.

On comprend donc toute l'attention qui y est portée.

Pratiquement, et compte tenu de la façon de développer et d'exploiter les sites on distingue les conséquences:

- liées au développement de la bulle ; la montée de pression peut compenser la baisse liée à l'augmentation régulière des prélèvements et améliorer ainsi la productivité des autres ouvrages, mais dans le cas de la remontée du niveau réservoir jusqu'en surface l'inondation de certaines zones est envisageable ;

- liées aux mouvements cycliques; de fortes évolutions annuelles avec un minimum très marqué peuvent conduire à l'assèchement temporaire de certaines sources ou à l'arrêt de la production de certains puits. Ceci peut être très gênant dans le cas où ce minimum intervient avec un retard de quelques mois par rapport à la pression du stockage c'est-àdire pendant l'été au moment des forts prélèvements.

Un nouveau projet ne peut donc être accepté qu'à deux conditions :

- des études aussi complètes et crédibles que possible doivent prouver que les perturbations à attendre sont selon toutes probabilités parfaitement acceptables car sans conséquence dommageable pour les utilisateurs ;

- un dispositif négocié de contrôle doit être défini, permettant de suivre en temps réel les évolutions effectives constatées et de prendre les mesures correctrices éventuellement nécessaires au niveau de l'exploitation du stockage. Ceci suppose également que les acteurs s'entendent sur la façon de caractériser l'état initial du système.

Nous nous intéresserons ici au premier aspect qui constitue le point central du dossier de demande en autorisation de stockage, le deuxième concernant le dispositif, intervenant davantage au niveau de son instruction comme sujet de négociation.

\section{DÉMARCHE MÉTHODOLOGIQUE DES ÉTUDES D'IMPACT}

Afin de répondre à ce genre d'interrogations des études très poussées sont de plus en plus indispensables. Les figures illustrant la démarche présentée ci-dessous sont issues des travaux liés au projet des Landes-de-Siougos. On peut résumer les étapes principales de la manière suivante

\subsection{Données et interprétation}

\subsubsection{Géologie régionale}

Constitution d'une description géologique régionale la plus complète possible par recours à des spécialistes extérieurs reconnus complétant et précisant l'information disponible 
dans le domaine public. Il convient alors de caractériser la géométrie et la pétrophysique (caractéristiques des roches) de toutes les zones susceptibles d'être influencées même marginalement par le stockage c'est-à-dire horizontalement une surface de l'ordre du millier de $\mathrm{km}^{2}$ et verticalement quelques centaines de mètres de terrains entourant le niveau réservoir. La géométrie des failles pouvant introduire des ruptures de continuité ou des mises en communication est à ce stade très importante. On définit généralement une dizaine de sous-niveaux principaux, qui couvrent chacun une partie de la zone d'étude et peuvent changer latéralement de caractéristiques (sables devenant des marnes par exemple). Les zones extérieures à ce premier ensemble réclament, au moins dans un premier temps, une description plus qualitative que quantitative. Elles peuvent être ensuite révaluées en fonction de l'impact simulé.

\subsubsection{Schéma hydraulique général}

Le schéma d'écoulement régional est indispensable à la prévision future de l'impact (fig. 1). En effet la compréhension des zones d'alimentation, des zones exutoires, du niveau naturel des pressions participe à la quantification de l'étendue et de l'efficacité des barrières naturelles aux écoulements (écrans horizontaux ou verticaux).

\subsubsection{Prélèvements}

Un bilan précis des prélèvements réalisés par les différents utilisateurs est également indispensable pour interpréter les évolutions de pression notées sur les puits de la zone concernée et être en mesure de quantifier plus précisément les caractéristiques des roches.

\subsubsection{Analyses}

Une série très complète d'échantillonnages et d'analyses est nécessaire pour la caractérisation hydrochimique des eaux de l'ensemble des aquifères concernés et fournir l'image initiale.

Les analyses réalisées sur le site (minéralisation, oligoéléments, hydrocarbures, sulfures, radioéléments, bactériologie) serviront de référence pour évaluer l'impact physico-chimique du stockage.

Les analyses réalisées sur d'autres ouvrages sont très importantes comme support aux études sur les schémas d'écoulement naturel dans les aquifères. Elles permettent de confirmer ou au contraire d'infirmer les communications entre zones séparées par des failles par exemple, ou d'apprécier la distance par rapport aux alimentations naturelles (analyse de tritium ou des isotopes ${ }^{14} \mathrm{C}$ et ${ }^{18} \mathrm{O}$ ).

A ce stade cependant de nombreuses hypothèses peuvent subsister en parallèle et c'est le rôle de l'étape suivante de tenter de répondre au plus grand nombre possible d'interrogations en particulier par la quantification des conséquences des hypothèses émises.

\subsection{Outil de simulation numérique}

\subsubsection{Préparation}

Il convient, à partir de l'ensemble des éléments précédents, d'effectuer un certain nombre de choix qui seuls permettent la transcription numérique et les calculs.

En particulier un regroupement logique de certains sousniveaux de caractéristiques voisines s'avère généralement nécessaire pour ne pas multiplier inutilement les couches du modèle et donc le nombre de mailles et la lisibilité des résultats (exemple fig. 2). On peut également faire le choix de ne

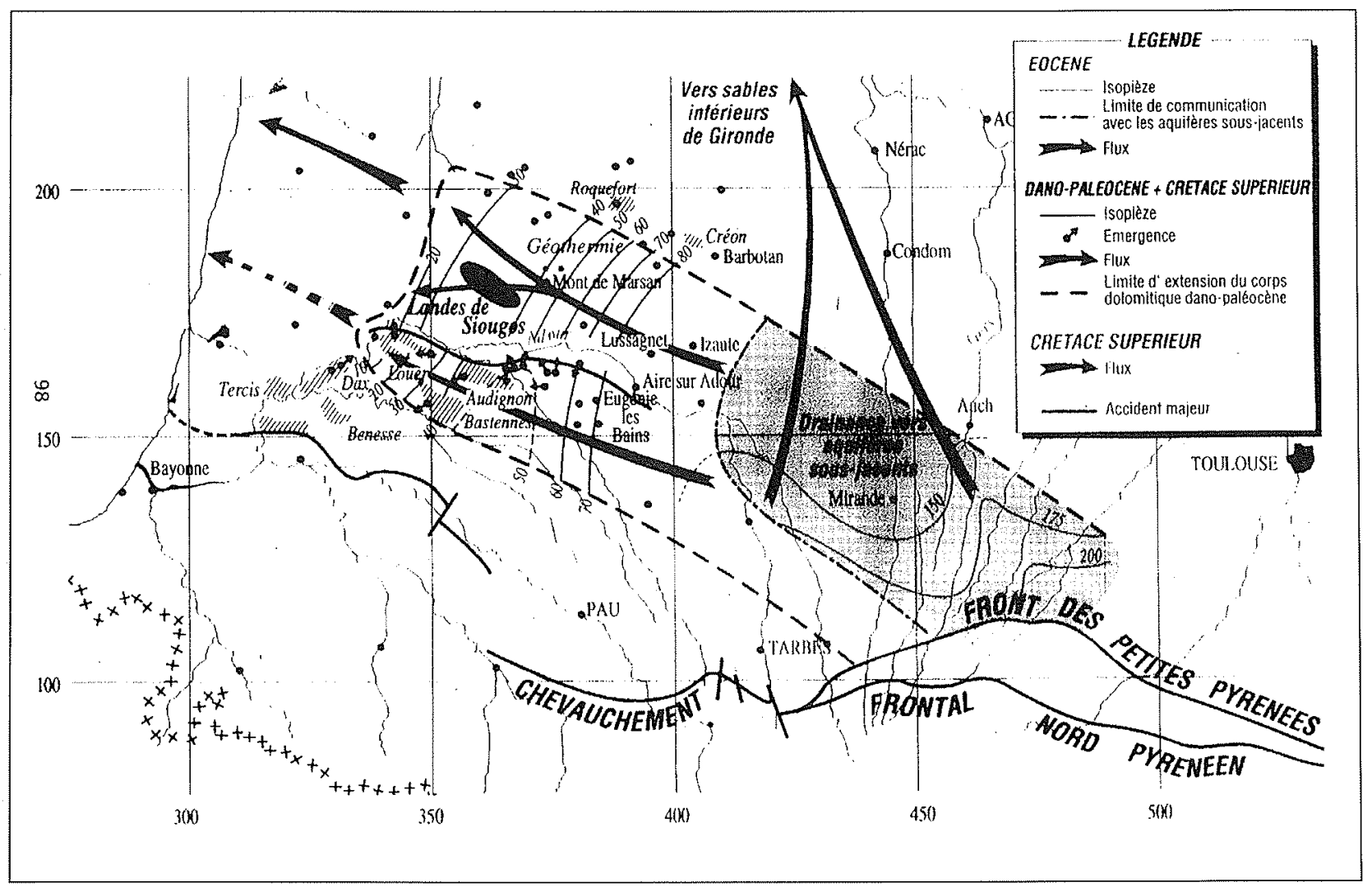

1. Schéma d'écoulement de la nappe profonde d'Aquitaine. 


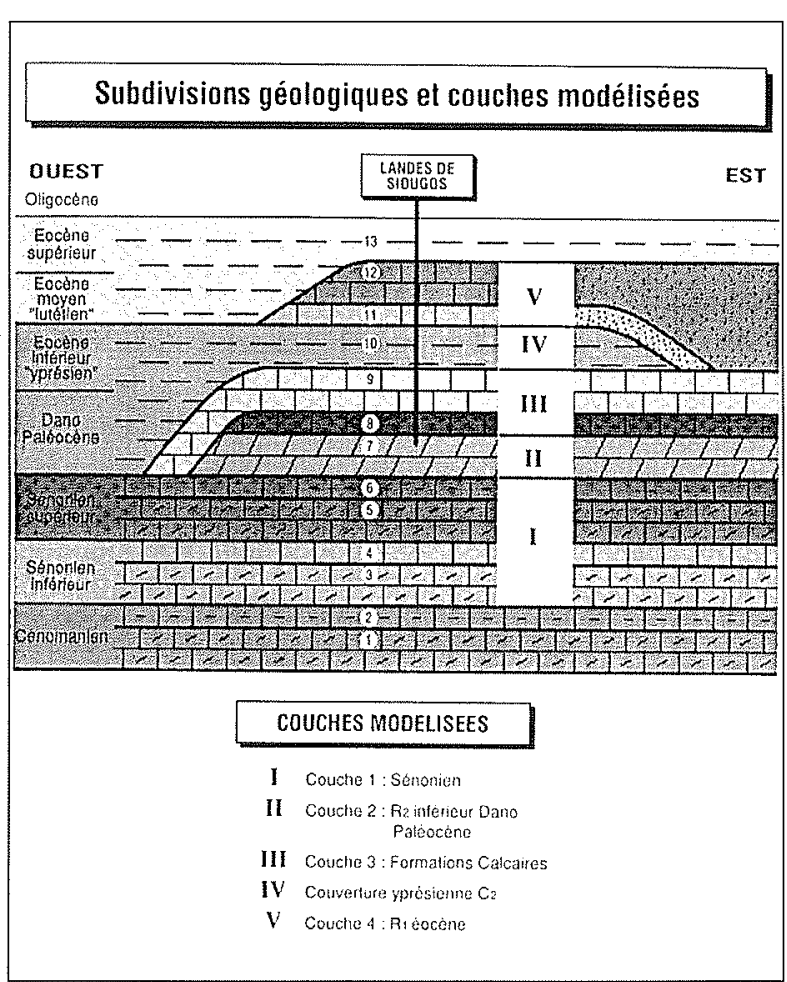

2. Subdivisions géologiques et couches modélisées.

pas détailler certaines zones dont on considère le rôle comme marginal : il faudra alors veiller à la validation de cet a priori.

Les alimentations et exutoires sont transformées en conditions à «pression imposée » ou « débit imposé ».

Le modèle utilisé étant généralement monophasique eau afin d'obtenir des temps de calcul beaucoup plus courts, les débits de gaz des différents stockages existants ou à venir sont transformés en débit d'eau équivalent par un modèle simplifié approprié.

\subsubsection{Calage}

La première étape de toute simulation raisonnable consiste à utiliser l'information dynamique connue (débits, pressions sur certains puits) pour vérifier la précision de l'information géologique entrée et quantifier de manière plus précise certains paramètres comme la transmissivité verticale entre couches ou les communications entre différents points par l'intermédiaire d'une simulation en régime «transitoire» (exemple fig. 3).

Une information précieuse à utiliser est, en plus, la connaissance du profil géographique naturel des pressions encore appelé "piézométrie " de la nappe. Outre son apport quant à la compréhension des mécanismes hydrauliques, sa restitution par le modèle est un élément-clef de sa fiabilité aux yeux des experts hydrogéologiques. Cet équilibre naturel met des centaines d'années à se réaliser quand on impose des conditions aux limites à partir d'un instant donné, en raison des vitesses d'écoulement très faibles de l'eau dans le sous-sol (quelques centimètres à quelques mètres par an). Aussi une adaptation du modèle d'écoulement est nécessaire pour obtenir ce régime "permanent » en quelques itérations.

Une difficulté peut intervenir lorsque les prélèvements sont à la fois anciens, très évolutifs et que leur importance devient prépondérante en comparaison avec les bilans de nappe. La définition d'un régime permanent devient alors délicate et peut conduire au calage d'un transitoire long (plu-

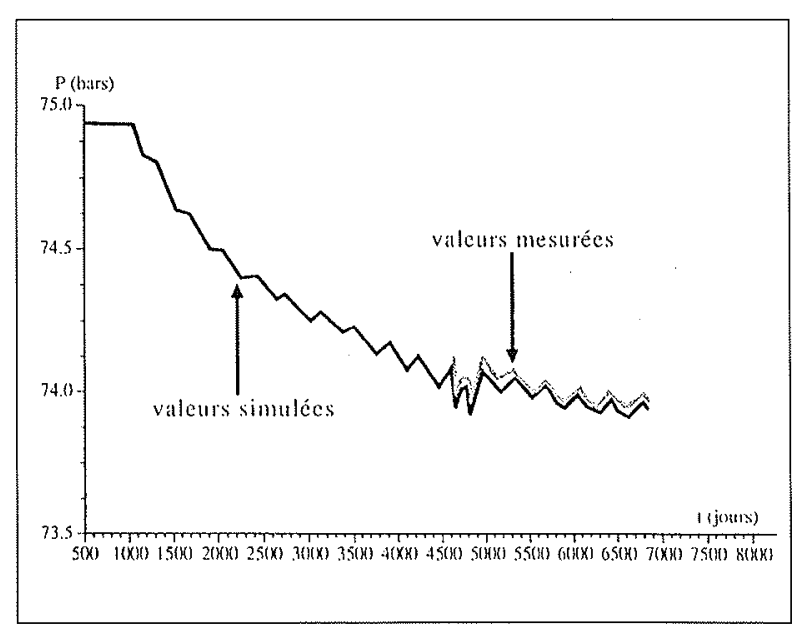

3. Pressions en eau mesurées et simulées au puits LS04 (R2).

sieurs dizaines d'années) beaucoup plus coûteux en efforts pour le chargé d'études.

Au travers de la description de ces deux premières étapes de l'utilisation du simulateur, le programme d'écoulements diphasiques MULTI développé au Gaz de France, on voit que déjà deux fonctionnalités particulières sont nécessaires: - la présence de sous-maillages qui permet de simuler une zone très étendue avec une bonne précision aux points importants et un nombre de mailles restreint (exemple fig. 4) ; - le calcul du régime permanent pour simuler l'état initial des aquifères que l'on couple au régime transitoire. Celui-ci démarre en même temps que les premiers prélèvements simulés. Si ces derniers sont très anciens et assez bien stabjlisés, ils pourront être intégrés au régime permanent.

Une fois l'ensemble de ces calages réalisé, la prévision de l'impact lié au futur stockage devient possible.

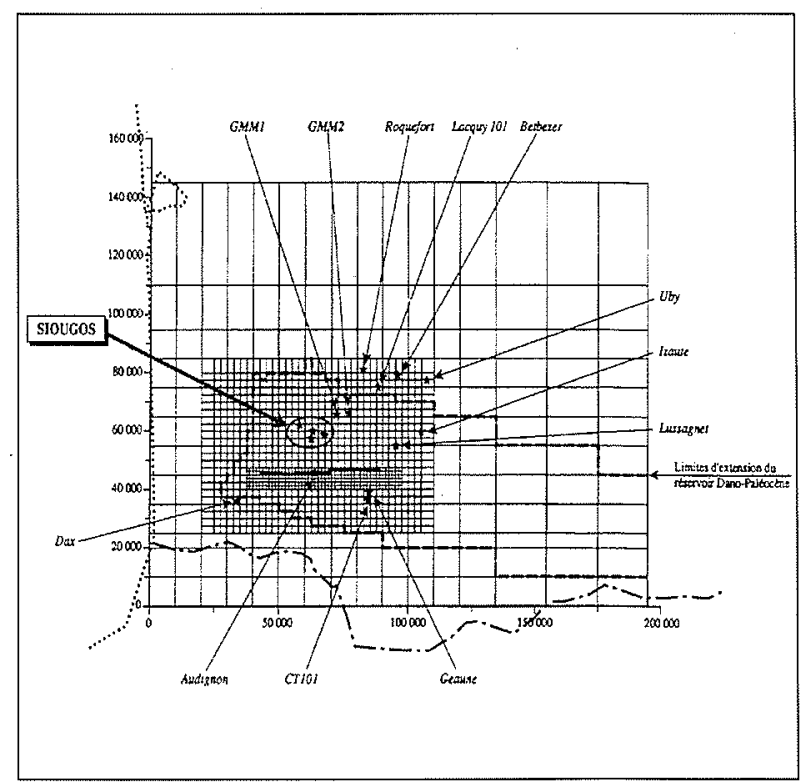

4. Landes de Siougos. Modèle géologique régional. Maillage et points remarquables. 


\subsubsection{La prévision d’impact hydrodynamique}

Il s’agit de la partie la plus facile à réaliser. L'historique le plus vraisemblable de développement puis d'exploitation du stockage est alors simulé.

La partie développement tient compte des besoins de modulation prévisibles et des limitations locales propres au stockage (débordement, pression maximale).

La partie exploitation considère un cyclage annuel sur une période de 50 ans de tout le volume utile disponible ce qui est bien sûr pessimiste.

\subsubsection{L'impact en pression}

Dans les zones profondes, la conséquence visible de la mise en service du stockage va être une évolution de la pression ou du niveau d'eau dans les puits existants.

Les résultats obtenus seront visualisés sous forme:

- de cartes de variations de pression par rapport à l'origine à différentes dates (par ex. 10 ans, 20 ans et 45 ans après le début du remplissage sur la fig. 5) et pour différentes couches;

- d'évolution au cours du temps de la pression en certains points sensibles. Un exemple typique en est donné par la figure 6 sur laquelle on observe une montée progressive lors du développement puis un retour vers l'équilibre initial à rythme très lent.

D'une manière générale, on considère qu'une montée de la pression inférieure à 1 bar soit $10 \mathrm{~m}$ de niveau d'eau dans les zones profondes ne demande généralement pas de mesure particulière et que au-delà il faut regarder plus en détail. Pour la variation annuelle quelques mètres peuvent être gênants mais heureusement l'amortissement dû aux terrains et à l'eau elle-même la rend souvent imperceptible audelà de $10-15 \mathrm{~km}$.

\subsubsection{L'impact en débit}

La quantité d'eau traversant certaines frontières au cours du temps est également un bon indicateur de l'impact du stockage. On pourra prendre par exemple un périmètre quelconque entourant le stockage à une certaine distance.

Pour l'étude de l'impact au niveau des affleurements plusieurs approches sont possibles:

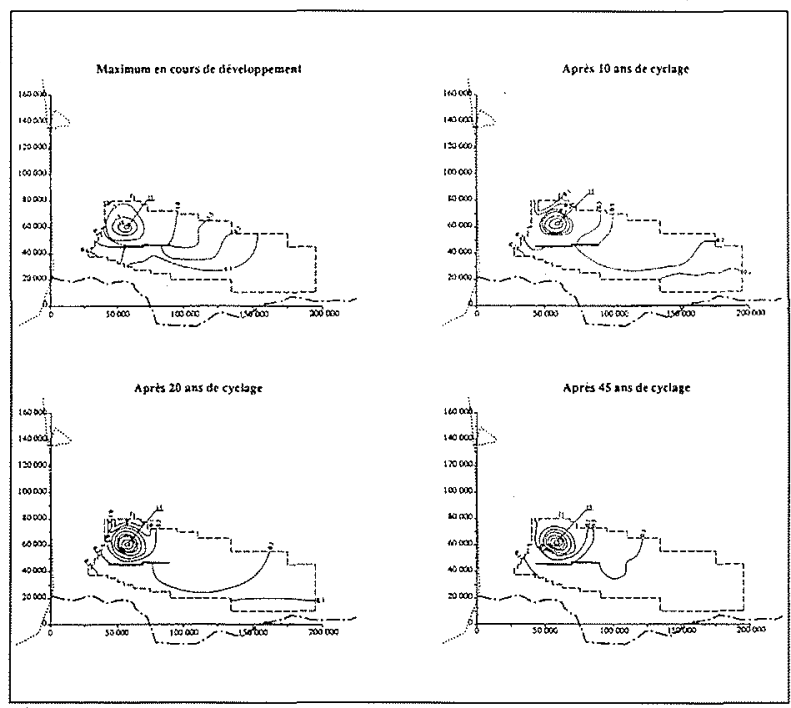

5. Impact hydrodynamique prévisionnel dans le $\mathbf{R} 2$ inférieur (bar).

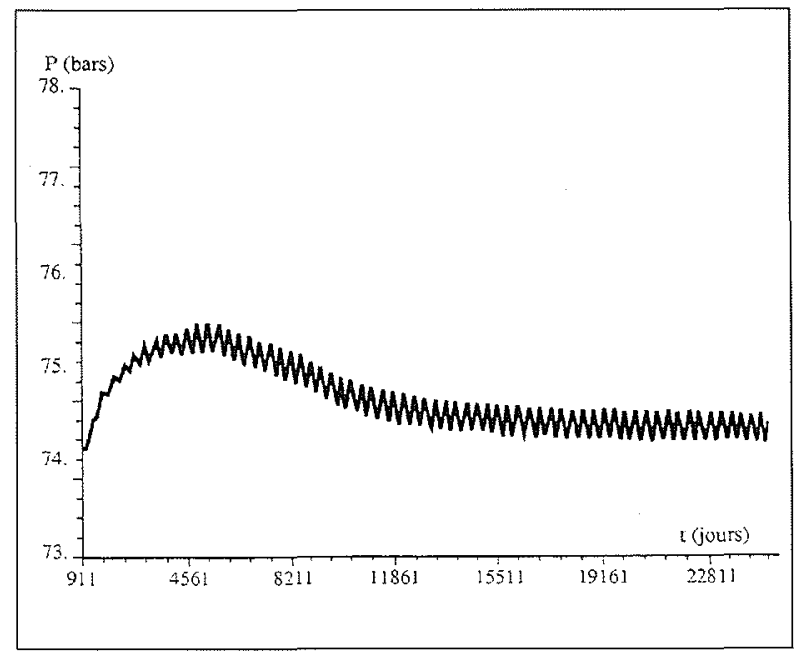

6. Evolution prévisionnelle de la pression à $10 \mathrm{~km}$ au sud, avant la ride d'Avignon.

- calcul de l'impact en pression en considérant cette «frontière » comme fermée. On obtient alors un majorant mais bien peu réaliste.

- calcul du débordement engendré par le stockage indépendamment des prélèvements locaux effectués et en faisant l'hypothèse que la zone concernée fonctionnait déjà en exutoire. On obtient alors un majorant d'un éventuel effet négatif.

Bien souvent, dans le cas d'émergences déjà largement exploitées on obtient en fait le débit supplémentaire qu'il sera possible de prélever à piézométrie constante.

\subsection{Discussion}

La base de connaissances et l'outil de simulation associé constituent une base évolutive. L'acquisition de données complémentaires ou la production d'études de synthèses supplémentaires peuvent infléchir dans une certaine mesure la vision initiale de l'objet d'étude et conduire à une réévaluation des conséquences prévisibles. Néanmoins, la prise en compte du plus grand nombre possible d'interactions réellement observées, lors de la phase de calage, est un garde-fou solide pour avancer les bons ordres de grandeur lors de la phase prévision. Par ailleurs, ce type d'outil permet toujours de tester les pires hypothèses dès lors qu'elles ne contredisent pas le calage réalisé et qu'elles sont conformes aux observations géologiques disponibles. Associer une probabilité est alors du ressort de l'expert hydrogéologue et définir le dispositif de surveillance celui de l'ensemble des acteurs concernés.

\section{$\mathrm{V}$ CONCLUSION}

L'utilisation rationnelle des nappes profondes constitue un enjeu très important pour l'avenir. La grande diversité et la qualité des travaux réalisés pour une insertion réussie des stockages de gaz naturel participe de manière très significative à l'amélioration des connaissances concernant la géologie et la dynamique des écoulements souterrains des bassins sédimentaires français. L'élargissement de ce type de démarche sur toute la surface des bassins concernés et incorporant toutes les mesures disponibles apparaît nécessaire à l'objectif final recherché. 